\title{
Multi-Resolution Analysis of Wavelet Like Soliton Solutions of KdV Equations
}

\author{
B. Bhosale, Member, IACSIT, and A. Biwas
}

\begin{abstract}
Many physical phenomena are described by nonlinear partial differential equations. These equations have soliton solutions which exhibit wavelet features called wavelet like solitons. Such wavelet like solitons have expansions in Gaussian family wavelets. In this work, using the fact that the wavelet like soliton has Gaussian representation, multiresolution analysis which is based on wavelets is carried out to obtain better approximation with the application of waveletGalerkin and wavelet-Petrokov-Galerkin methods for soliton solution of Korteweg-de Vries equation which appears in the study of waves in shallow water in the fluid dynamics. In the end, experimental data processing employing Gaussian representation of soliton solution is discussed.
\end{abstract}

Index Terms -Wavelet like solitons, gaussian representation, wavelet decomposition, data processing.

\section{INTRODUCTION}

Multi-resolution analysis (MRA) uses wavelet functions as basis with an objective to specify the signal as a collection of its successive approximations. This approximations are of different resolutions, whence the name multi-resolution analysis. The term wavelet or the phrase wavelet analysis was first coined by J. Morlet [1]. Earlier, wavelets were used in electrical engineering. The major breakthrough occurred due to D. Gabor [2], who introduced the Windowed Fourier Transform (WFT) for the local spectral analysis of radar signals that actually laid pathway for use of wavelets from electrical engineering to mathematical physics. Due to the limitation of WFT, where the localization is attained due to fast decaying window functions, the scheme called, Wavelet transform (WT) with a wide window for low frequency signals and a narrow window for high frequency signals was introduced and formalized later by Grossman and Morlet [1], Daubechies [3] and many others. Recently, wavelet transform has been emerged as the most effective tool for signal processing and image analysis especially when the signals are random and comprised of fluctuations of different scales. Wavelets have been used in signal processing, problems involving singular potentials in quantum mechanics, in discussions concerning q-algebras, and even in nuclear structure studies [4]. In wavelet inspired approach, the sets of 'wavelets' are employed to approximate signals because of their Gaussian form. The beauty of the wavelet analysis lies in its predominant property of self-similarity that makes wavelet as a powerful tool for analyzing fractal like patterns. Since

Manuscript received March 12, 2013; revised May 20, 2013.

B. Bhosale is with the S.H.Kelkar College, affiliated to University of Mumbai, India (e-mail: bn.bhosale@rediffmail.com).

A. Biwas is with the Delaware State University in Dover, USA. the soliton-like solutions have infinite extent, it requires rather appropriate compactly supported basis functions to investigate such structures than the traditional nonlinear tools (inverse scattering, group symmetry, functional transforms) that are not always applicable. Such structures/patterns generally have finite space-time extension and a multi-scale structure. Multi-resolution analysis that uses wavelet functions could be therefore a natural useful method for the construction of such nonlinear bases. This motivated us to employ the wavelet methods to analyze wavelet like soliton solutions of .Korteweg-de Vries equation $(\mathrm{KdV})$ that appears in the study of waves in shallow water in the fluid dynamics. We have in [5]-[7], carried out extensively the wavelet analysis of solitons arising as solutions of Non-linear Schrodinger Equation (NLS), Sine-Gordon equation (SG).

\section{MATHEMATICAL PRE-REQUISITES}

We need some mathematical formulations that are relevantly useful in the present work.

\section{A. Wavelet Transforms}

Practically wavelet transform is a convolution of the signal with a family of functions obtained from a basic wavelet by shifts and dilations. In precise terms and notations, the classical wavelet transform, also called as Continuous Wavelet transform (CWT) [8], is a decomposition of a function, $f(x)$, with respect to a basic wavelet $\psi(x)$, given by the convolution of a function with a scaled and translated version of $\psi(x)$

$$
W_{\psi}(a, b)[f]=|a|^{-1 / 2} \int f(x) \psi^{*}\left(\frac{x-b}{a}\right) d x
$$

The functions, $f$ and $\psi$ are square integrable functions and $\psi$ satisfies the admissibility condition: $C_{\psi}=$ $\int \frac{|\widehat{\psi}(\omega)|^{2}}{|\omega|} d \omega<\infty . C_{\psi}$ is called admissibility constant. The subscript ' $*$ ' denotes complex conjugation, ' $a$ ' is the scale parameter, $a>0$, ' $b$ ' is the translation parameter. The term $1 / \sqrt{|a|}$ is the energy conservative term that keeps energy of the scaled mother wavelet equal to the energy of the original wavelet. The function $f(x)$ can be recovered by the reconstruction formula called Inverse transform:

$$
f(x)=\frac{1}{c_{\psi}} \iint W_{\psi} f(a, b) \frac{1}{\sqrt{|a|}} \psi\left(\frac{x-b}{a}\right) \frac{d a d b}{a^{2}}
$$

where the admissibility constant, $C_{\psi}>0$. Therefore, any function with compact support which satisfies above requirement can be successfully used as a basic wavelet. 
Furthermore, with the substitution, $f(x)$ as the inverse Fourier Transform $f(x)=\frac{1}{2 \pi} \int_{-\infty}^{\infty} \exp (i \omega x) \hat{f}(\omega) d \omega$ in the definition of wavelet transform (1), we immediately get the spectral representation as

$$
W_{\psi}[f(x)](a, b)=\frac{1}{2 \pi}|a|^{1 / 2} \int_{-\infty}^{\infty} \exp (i \omega b) \overline{\hat{\psi}(a \omega)} \hat{f}(\omega) d \omega .
$$

\section{B. Discretization}

The convenient way for numerical implementation of WT is its discretised version, called Discrete Wavelet transform (DWT). With $a$ and $b$ as scale and translation parameters, taking scale $a: a=a_{0}^{m}$ and the translation $b: b=n b_{0} a_{0}^{m}$, where $a_{0}$ and $b_{0}$ are the discrete scale and translation step sizes, respectively, the DWT is given by [8]

$$
\begin{aligned}
& W_{\psi}(m, n)[f]=\frac{1}{\sqrt{a_{0}^{m}}} \int_{-\infty}^{\infty} f(x) \psi\left(\frac{x-n b_{0} a_{0}^{m}}{a_{0}^{m}}\right)= \\
& \frac{1}{\sqrt{a_{0}^{m}}} \int_{-\infty}^{\infty} f(x) \psi\left(a_{0}^{-m} x-n b_{0}\right) d x
\end{aligned}
$$

For $a_{0}=1$, the reconstruction of $f(x)$ is given by

$$
f(x) \approx k \sum_{m=0}^{\infty} \sum_{n=0}^{\infty}\left[W_{\psi} f(m, n)\right] a_{0}^{-\frac{m}{2}} \psi\left(a_{0}^{-m}-n b_{0}\right)
$$

where $k$ is the constant that depends upon the redundancy of the basic wavelet and the lattice combination which is ignored in many applications and $\tilde{\psi}_{m, n}$ is wavelet dual of $\psi_{m, n}$.

\section{KORTEWEG-DE VRIES EQUATION}

The generalized Korteweg-de Vries equation with timedependent damping and dispersion [9]:

$$
q_{t}+q^{n} q_{x}+a(t) q+b(t) q_{x x x}=0
$$

The first term of the equation is the evolution term, the second term represents the nonlinear term, while the third term is the linear damping with a time-dependent coefficient $a(t)$ while the fourth term is the dispersion term with timedependent coefficient $b(t)$. In (6), $a, b \in R$ while $n \in Z^{+}$.

The solitary wave solution to (6) is given by

$$
q(x, t)=\frac{A(t)}{\cosh ^{p}[B(t)(x-v(t) t]}
$$

where $A$ represents the amplitude of the soliton, while $B$ is the inverse width of the soliton and $t$ represents the velocity of the soliton. Thus, for $p=\frac{2}{n}$, without loss of generality, (7) takes the form

$$
q(x, t)=\frac{A}{\cosh ^{\frac{2}{n}}[B(x-v t)]}=A \operatorname{sech}^{\frac{2}{n}}[B(x-v t)]
$$

when $n=1$,

$$
q(x, t)=A \operatorname{sech}^{2}[B(x-v t)]
$$

The same can be written as

$$
q(x, t)=q(s) \text { with } s=x-v t
$$

\section{Mathematical ANALYSiS}

Most often the signals have a Gaussian form and display self-similar fractal like patterns. We have from [4], the soliton-like solution, $u(x, t)=u(s)$ with $s=x-v t$ has expansion in a Gaussian family of wavelets $\psi(s)=N e^{Q(s)}$, where $Q(s)$ is a polynomial and $\mathrm{N}$ the normalization constant. In particular, if we choose $Q(s)=-i s-\frac{s^{2}}{2}$, we obtain a very particular wavelet with the support mainly confined in the $(-1,1)$ interval, namely $\psi(s)=$ $\exp \left[-i s-\frac{s^{2}}{2}\right]^{\pi^{1 / 4}}$.

We shall use this fact in the development of procedure for approximating the soliton solution through wavelet decomposition and in the further application of experimental data processing.

We consider the most celebrated generalized $\mathrm{KdV}$ equation obtained from (6) with $n=1$

$$
q_{t}+q q_{x}+\mu q+v q_{x x x}=0
$$

The equation (11) can be written in differential operator form:

$$
\widehat{L}(q(x, t))=0, \text { where } \widehat{L} \equiv \frac{\partial}{\partial t}+q \frac{\partial}{\partial x}+q+\frac{\partial^{3}}{\partial x^{3}}
$$

To apply the wavelet method or more appropriately, the wavelet-Galerkin method, the solution is decomposed with respect to the wavelet basis

$$
q(s)=q(x, t)=\sum_{j, k} C_{j, k}(t) \psi_{j, k}(x)
$$

where $C_{j, k}(t)$ are the time dependent wavelet coefficients and $\psi_{j, k}(x)$ is admissible function/basic wavelet to be taken as

$$
\psi_{j, k}(x)=h^{-j / 2} \psi\left(h^{-j} x-k\right) .
$$

This is a discrete expansion or wavelet decomposition of soliton solution $q(s)$ in terms of integer translations $(k)$ of $\psi$ which provide the analysis of localization, and in terms of dyadic dilations $\left(h^{j}\right)$ of $\psi$, which provide the description of different scales.

Substituting the decomposition (13) into (12), it yields the system of equations

$$
\sum_{j, k} C_{j, k}(t) \hat{L} \psi_{j, k}(x)=0 .
$$

By scalar multiplication $\int d x \bar{\psi}_{l, m}$, where $\bar{\psi}_{l, m}$ is dual wavelet, we obtain the orthogonal system of compactly supported wavelets $\Omega_{m k}^{l j} \equiv \int d x \bar{\psi}_{l, m} \hat{L} \psi_{j, k}(x)$.

The system of (14) becomes

$$
\sum_{j, k} \Omega_{m k}^{l j} C_{j, k}=0
$$

This is a system of ordinary differential equations in the wavelet coefficients $C_{j, k}$. 
For the orthogonal Daubechies wavelets with compact support, only the matrix elements $\Omega_{m k}^{l j}$ with the basic functions of the same scale $l=j$ are different from zero. Thus, (15) provides a sparse structure of non-linear system suitable for numerical implementation.

The main component of the wavelet-Galerkin solution is the evaluation of the matrix elements $\Omega_{m k}^{l j}$ of the differential operators in wavelet basis $\psi_{j, k}$.

For this purpose, the analytically determined wavelets such as Mexican hat, Morlet wavelets are employed.

The wavelet -Galerkin scheme for (11) consists in substitution of discrete wavelet decomposition of the solution $q(x, t)=\sum_{j, k} C_{j, k}(t) \psi_{j, k}(x)$ in to (11), followed by the projection of the result onto orthogonal basis of $\psi_{l . m}$

$$
\begin{aligned}
& \int d x \bar{\psi}_{l, m}(x)\left[\dot{C}_{j, k}-C_{s, r} \psi_{s, r}(x) C_{j, k} \frac{d}{d x}+\mu C_{j, k}+\right. \\
& \left.v C_{j, k} \frac{d^{3}}{d x^{3}}\right] \psi_{j, k}(x)=0
\end{aligned}
$$

For the orthogonal Daubechies wavelets with compact support, this gives a system of nonlinear ordinary differential equations with unknown wavelet coefficients $C_{j, k}$ depending on time $t$ only

$$
\dot{C}_{l, m}-\Omega_{m r k}^{l s j} C_{j, k}+\mu \Omega_{1, m k}^{l j} C_{j, k}+v \Omega_{m k}^{l j} C_{j, k}=0
$$

where the matrix elements are

$$
\begin{gathered}
\Omega_{m r k}^{l s j}=\int d x \bar{\psi}_{l, m}(x) \psi_{s, r}(x) \frac{d}{d x} \psi_{j, k}(x), \\
\Omega_{1, m k}^{l j}=\int d x \bar{\psi}_{l, m}(x) \psi_{j, k}(x) \\
\Omega_{m k}^{l j}=\int d x \bar{\psi}_{l, m}(x) \psi_{s, r}(x) \frac{d^{3}}{d x^{3}} \psi_{j, k}(x) .
\end{gathered}
$$

The direct integration in the matrix elements is numerically unstable for the irregularity of the basic functions $\psi(x)$. However, they can be evaluated analytically. When all coefficients of (18) are known, the system of ordinary differential equations (17) can be solved numerically by an implicit or explicit method.

In the simplest case of an explicit scheme we have $C_{l, m}(t+\tau)=C_{l, m}(t)+\tau\left[\Omega_{m r k}^{l s j} C_{j, k}(t) C_{r, s}(t)+\right.$ $\left.\mu \Omega_{1, m k}^{l j} C_{j, k}+v \Omega_{m k}^{l j} C_{j, k}(t)\right]$, where $\tau$ is a time step of integration.

The evaluation of the matrix elements of all differential operators is provided by the knowledge of connection coefficients- the matrix elements of those operators in the basis of wavelet scaling function $\varphi(x) \quad \Lambda_{k_{1 \ldots k_{n}}}^{\left(d_{1} \ldots d_{n}\right)}=$ $\int d x \varphi_{k_{1}}^{\left(d_{1}\right)} \ldots \varphi_{k_{n}}^{\left(d_{n}\right)}$, where the superscripts of the parentheses stand for the order of differentiation. Then all the terms with the wavelet basic functions $\psi$ are evaluated by the substitution $\psi(x)=\sqrt{2} \sum g_{n} \varphi(2 x-1)$. The general method of evaluation of connection coefficients is presented in [10].

Alternative scheme of evaluating the system of differential equations is provided by modified methodWavelet-Petrokov-Galerkin method (WPG) [11], where we make the substitution $\psi_{j, k}(x)=h^{-j / 2} \psi\left(h^{-j} x-k\right)$ in the expression (16) to write

$$
\begin{aligned}
& \sum_{j, k} \int d x h^{-j / 2} \psi\left(h^{-j} x-m\right)\left[\frac{\partial}{\partial t} C_{j, k}-C_{s, r} h^{-j / 2} \psi\left(h^{-j} x-\right.\right. \\
& \left.r) C_{j, k} \frac{d}{d x}+\mu C_{j, k} \frac{d}{d x}+v C_{j, k} \frac{d^{3}}{d x^{3}}\right] h^{-j / 2} \psi\left(h^{-j} x-k\right)=(19)
\end{aligned}
$$

Introducing the change of variable $y=h^{-j} x-k$, the expression (19) becomes

$$
\begin{array}{r}
\sum_{j, k} a(k) \frac{d C_{j, k}}{d t}+h^{-3 j / 2} \sum_{s, r} \sum_{j, k} b(l, k) C_{s, r} C_{j, k}+ \\
\mu h^{-j / 2} \sum_{j, k} a(k) C_{j, k}+v h^{-3 j} \sum_{j, k} d(k) C_{j, k}=0
\end{array}
$$

where $a(k)=\int d y \psi(y) \bar{\psi}(y-k)$,

$$
\begin{gathered}
b(l, k)=\int d y \frac{d \psi(y)}{d y} \bar{\psi}(y-k), \\
c(k)=(-1)^{\beta} \int d y \frac{d^{\alpha} \psi(y)}{d y} \frac{d^{\beta} \bar{\psi}(y-k)}{d y} .
\end{gathered}
$$

The unknown coefficients $C_{j, k}$ are determined from the system of ordinary differential equations written in matrix form:

$$
\frac{d}{d t} L C+C^{T} M C+N C+T C=0
$$

where

$$
\begin{gathered}
C=C_{j, k}, L(l, k)=a(l-k), \\
M(l, k, s)=h^{-3 j / 2} b(l-k, l-s), \\
N(l, k)=\mu h^{-j / 2} a(l-k), T(l, k)=v\left(h^{-3 j} c(l-k) .\right.
\end{gathered}
$$

Note that the unknown coefficients are only the time dependent. By Trapezoidal rule $\frac{d C}{d t}=\frac{C^{n+1}-C^{n}}{\Delta t}$, where $\Delta t=t_{n+1}-t_{n}$, the time interval.

The equation (21) becomes

$$
L\left(\frac{C^{n+1}-C^{n}}{\Delta t}\right)+C^{T} M C+N C+T C=0 .
$$

Now setting $G(C)=C^{T} M C+N C+T C$, we have from (22)

$$
L\left(C^{n+1}-C^{n}\right)+\frac{G\left(C^{n+1}\right)+G\left(C^{n}\right)}{2} \Delta t=0
$$

This algebraic equation can be finally solved by Newton's iterative method using the recursive construct

$$
U^{n+1}=U^{n}-\frac{f\left(U^{n}\right)}{f^{\prime}\left(U^{n}\right)}, n=0,1,2, \ldots
$$

The solution thus obtained by approximation process can be eventually compared with the exact solution obtained from (9) $q(x, t)=A \operatorname{sech}^{2}[B(x-v t)]$ computed at different positions depending on time.

\section{EXPERIMENTAL DATA PROCESSING}

In experimental data processing, the central problem either in one dimensional or multi-dimensional set up is the 
separation of two or more signals from a noisy background. Most often these signals have a Gaussian form which itself is a wavelet. Therefore, the wavelet analysis provides robust method in the presence of noise especially if taken as wavelet image of the Gaussian $\exp \left(-s^{2} / 2\right)$ with vanishing momenta wavelets $\frac{d^{n}}{d s^{n}} \exp \left(-s^{2} / 2\right)$ known analytically. The central idea here is to assume the Gaussian distribution representing the soliton solution as the best fit for the experimental data and take the wavelet image of this Gaussian as testing wavelet with appropriate analytically tested function as analyzing wavelet say.

Recalling that the wavelet like soliton solution of $\mathrm{KdV}$ has the Gaussian form, we can therefore assume that Gaussian function is the 'best fit' to describe the experimental data set to be processed as testing wavelet function. Then, the problem of fitting the distribution of Gaussian sources is to find the parameter set $\left(N^{k}, \sigma_{k}, s_{k}^{m}\right)_{k=1}^{M}$ that minimizes the difference

$$
F\left(N, \sigma, x^{m}\right)=f_{\exp }(x)-\sum_{k=1}^{M} \frac{N_{k}}{\sqrt{2 \pi \sigma_{k}^{2}}} \exp \left(-\frac{\left(x-x_{k}^{m}\right)^{2}}{2 \sigma_{k}^{2}}\right)
$$

Applying wavelet transform to (24) with some analytically tested basic wavelet, say, Mexican hat or Morlet wavelet, one can precisely locate the position of the sources $s_{k}^{m}$.

Let us start with the wavelet image of a single Gaussian representing the soliton, located without loss of generality at $s^{m}=0$.

$$
q_{\text {gauss }}(s)=\frac{N}{\sqrt{2 \pi \sigma_{k}^{2}}} \exp \left(-\frac{s^{2}}{2 \sigma_{\mathrm{k}}^{2}}\right), s=x-v t
$$

We need the wavelet images of the Gaussian with different vanishing momenta wavelets as analyzing wavelet where the first $\mathrm{m}$ family of vanishing momenta wavelets [12], of basic wavelets $\psi$ :

$$
g_{n}(s)=-1^{n+1} \frac{d^{n}}{d x^{n}} \exp \left(-s^{2} / 2\right), n>0
$$

which satisfies the condition

$$
\int d s s^{m} \psi(s)=0, \forall m, 0 \leq m<n, n \in Z
$$

The wavelet images of the Gaussian with vanishing momenta wavelet are therefore given by:

$$
W_{g_{n}}(a, b)\left[q_{\text {gauss }}\right]=\int \overline{\frac{1}{\sqrt{a}} g_{n}\left(\frac{s-b}{a}\right)} q_{\text {gauss }}(s) d s .
$$

The integrals in (26) can be evaluated if the Fourier representation (3)

$$
\frac{1}{2 \pi}|a|^{1 / 2} \int_{-\infty}^{\infty} \exp (i k b) \frac{W_{\psi}(a, b)[q}{\tilde{g}_{n}(a k)} \tilde{q}_{g a u s s}(k) d k
$$

where $\tilde{g}_{n}(k)=\sqrt{2 \pi}(i k)^{n} \exp \left(-k^{2} / 2\right)$.

Instead of evaluating integrals for each $n$ separately, we can evaluate it once for the Morlet wavelet

$$
\tilde{g}(\tau, k)=\sqrt{2 \pi} \exp \left(i k \tau-k^{2} / 2\right)
$$

and then take the $n$th derivative of $n$ with respect to the formal parameter $\tau$ at $\tau=0$ to obtain the wavelet image of $g_{n}$ family:

$$
\begin{gathered}
\tilde{g}_{n}(k)=\left.\left(\frac{d}{d \tau}\right)^{n}\right|_{\tau=0} \tilde{g}(\tau, k) \\
W_{g_{n}}(a, b)=\left.\left(\frac{d}{d \tau}\right)^{n}\right|_{\tau=0} W_{g(\tau)}(a, b)
\end{gathered}
$$

Substituting (28) instead of $\tilde{g}_{n}(k)$ into (29) and taking into account the Fourier image of Gaussian (25)

$$
\begin{aligned}
& \tilde{q}_{\text {gauss }}(k)=N \exp \left(-\frac{\mathrm{k}^{2} \sigma^{2}}{2}\right), \text { we arrive at } \\
& \begin{aligned}
W_{g_{n}}(a, b)\left[q_{\text {gauss }}\right] & =N \sqrt{\frac{a}{2 \pi}} \int d k \exp (i k(b-a \tau)- \\
\left.\frac{k^{2}}{2}\left(a^{2}+\sigma^{2}\right)\right) & =N \sqrt{a} \frac{\exp \left(-\frac{(b-a \tau)^{2}}{2\left(a^{2}+\sigma^{2}\right)}\right)}{\sqrt{a^{2}+\sigma^{2}}}
\end{aligned}
\end{aligned}
$$

for the wavelet image of a single Gaussian with respect to the analyzing wavelet, vanishing momenta wavelet $g_{n}$ in instant case (30).

To find the distribution parameters for the case of single Gaussian source we use the coefficients of its $g_{2}$ decomposition.

Equation (30) for $n=2$ for example leads to

$$
\begin{gathered}
W_{g_{2}}(a, b)\left[q_{\text {gauss }}\right]= \\
N a\left(\frac{a}{a^{2}+\sigma^{2}}\right)^{3 / 2}\left[1-\frac{b^{2}}{a^{2}+\sigma^{2}}\right] \exp \left(-\frac{b^{2}}{2\left(a^{2}+\sigma^{2}\right)}\right)
\end{gathered}
$$

Taking the derivative $\partial / \partial a$ of (32) at the central point $b=0$, we find the extremum of the $g_{2}$ coefficient at a scale $a_{m}=\sqrt{5 \sigma}$.

The value of the wavelet coefficient at the extremal point is therefore

$$
W_{g_{2}}\left(a_{m}, 0\right)\left[q_{\text {gauss }}\right]=\frac{N}{\sqrt{\sigma}} 5^{5 / 4} 6^{-3 / 2}=\frac{N}{\sqrt{a_{m}}}\left(\frac{5}{6}\right)^{3 / 2}
$$

Thus, performing the convolution (1) or for numerical implementation (4) with $g_{n}(s) \equiv \psi_{j, k}(x)=h^{-j / 2} \psi\left(h^{-j} x-k\right) \quad$ numerically and finding the maximum of the $g_{2}$ wavelet coefficient we obtain the dispersion and amplitude of the original distribution $q_{\text {gauss }}$ such that

$$
q_{\text {gauss }}=\sum_{j k} \tilde{\psi}_{j, k}<\psi_{j, k}, q_{\text {gauss }}>
$$

\section{REFERENCES}

[1] A. Grossman and J. Morlet, "Decomposition of hardy functions into square-integrable wavelets of constant shape," SIAM J. Math. Anal., vol. 15 , no. 4 , pp. $723-736,1984$.

[2] D. Gabor, "Theory of communication," in Proc. IEE, vol. 93, 1946, pp. 429-457.

[3] I. Daubechies, "Orthogonal bases of compactly supported wavelets," J. Comm. Pure. Apl. Math., vol. 41, pp. 909-996, 1988.

[4] A. Ludu and J. P. Draayer, "Solitons and wavelets: Scale analysis and Bases," J. Phys. Rev. Lett., vol. 10, pp. 2115-2125, 2000. 
[5] B. Bhosale and A. Biswas, "Wavelet analysis of optical solitons and its energy aspects," J. Mathematics in Engineering, Science and Aerospace, vol. 3, no. 1, pp. 15-27, 2012.

[6] B. Bhosale and A. Biswas, "Wavelet analysis of soliton interaction and its relation to probability distributions," J. Nonlinear Studies.

[7] B. Bhosale, A. Yildirim, and A. Biswas, "Modeling space-timevarying systems for analyzing solitons," J. Advanced Sciences, Engineering and Medicine, vol. 4, pp. 164-170, 2012.

[8] R. K. Young, Wavelet theory and its Applications, Kluwer Academic Publishers, Fourth Printing, 1995.

[9] A. Biswas, "Solitary wave solution for the generalized KdV equation with time dependent damping and dispersion," J. Commun Nonlinear Sci Numer Simulat, vol. 14, pp. 3503-3506, 2009.

[10] G. Beylkin, "On the representation of operators in bases of compactly supported wavelets," SIAM J. Numerical Analysis, vol. 6, no. 6, pp. 1716-1740, 1992.

[11] J. Villegas and J. Castano, "Wavelet-Petrov-Galerkin method for the Numerical Solution of the KdV Equation," J. Applied Mathematical Sciences, vol. 6, no. 69, pp. 3411-3423, 2012.

[12] J. M. Combes, A. Grossmann, and P. Tchamitchan, Wavelets, Berlin: Springer, 1989.

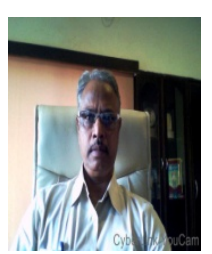

B. Bhosale comes from Maharashtra, India. He earned B.Sc (Hon) and M.Sc from and Ph. D degrees in mathematics from Shivaji University,

Kolhapur. He has worked as the associate professor in mathematics in the University of Mumbai and at present he is working as the principal of S. H. Kelkar (Undergraduate and Post graduate) college, affiliated to University of Mumbai. He has visited several countries as invited speaker. He has published 12 research papers in national/international journals and two books viz. Theory of Integral transformations of generalized functions (2007), Mathematical aspects of physical concepts and physical aspects of Mathematical concepts (2010), Discovery publishing house, New Delhi. His research area is integral transforms and its applications in signal/image processing. Currently he is working on wavelets and solitons. Dr. Bhosale is a member of Indian Mathematical Society (IMA), International Association of Computer Science and Information Technology (IACSIT). He has been awarded with Bharat Gaurav citation for his contribution in the field of higher education.

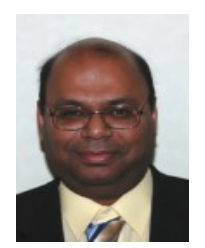

A. Biswas comes from West Bengal. He earned his B.S. (honors) degree from Saint Xavier's College in Calcutta, India, and his M.S. and M. Phil degrees in applied mathematics from the University of Calcutta. He earned M.A. and Ph.D. degrees in applied mathematics from the University of New Mexico, Albuquerque, USA, and a Ph.D. degree in applied mathematics from the University of Colorado, Boulder, USA. He is an assistant professor of Mathematics at Tennessee State University, Nashville, USA, and is currently working as an associate professor of Mathematics at Delaware State University in Dover, USA. His research interests include the theory of solitons. 